\title{
CONOCIMIENTO PARA LA INNOVACIÓN EN LA EMPRESA FAMILIAR hotelera. Caso: destino turístico Mazatlán
}

\author{
Ana María Larrañaga Núñez \\ amlarranaga2607@gmail.com \\ Anel Yadira Pérez Melo \\ aypmudomzt@hotmail.com \\ Universidad Autónoma de Occidente \\ Unidad Regional Culiacán
}

El objetivo de este trabajo es identificar la integración de conocimientos para la innovación de procesos y organizativa en la empresa familiar hotelera de Mazatlán, Sinaloa. El conocimiento es un recurso clave que procede de distintas fuentes y vínculos internos y externos y coadyuva con la innovación en un mercado globalizado. La descripción cuantitativa emana de los cuestionarios aplicados a cuatro empresas. Los hallazgos demuestran la realización de actividades de innovación, a excepción de la introducción de investigación y desarrollo externo; además, el conocimiento proviene de fuentes gratuitas e internas. Se concluye que estas empresas evitan riesgos para innovar por el costo que representa la compra de conocimiento.

Palabras clave: Gestión del conocimiento, vinculación del conocimiento, innovación, empresa familiar hotelera, destino turístico.

\section{KNOWLEDge FOR INNOVATION IN THE FAMILY HOTEL BUSINESS. Case: tourist destination Mazatlan}

The objective of this work is to identify the integration of knowledge for process and organizational innovation in the family hotel business of Mazatlan, Sinaloa. Knowledge is a key resource that comes from different sources and internal and external links and that contributes to innovation in a globalized market. The quantitative description emanates from the questionnaires applied to four companies. The findings demonstrate the realization of innovation activities except for the introduction of external research and development, in addition the knowledge comes from free and internal sources. It is concluded that these companies avoid risks to innovate because of the cost of buying knowledge.

Key words: Knowledge management, Knowledge linking, Innovation, Hotel family business, Tourist destination.

Fecha de recepción: 17 de enero de 2019. Fecha de aceptación: 15 de abril de 2019

CÓMO CITAR: Larrañaga, A. M. y Pérez, A. (2019). Conocimiento para la innovación en la empresa familiar hotelera. Caso: destino turístico Mazatlán. Dimensiones Turísticas, 3(4), 43-62. https://doi.org/10.47557/ZDIW3995 
$E^{\prime}$ conocimiento es un recurso clave en la hotelería para mantener ventajas competitivas en un mercado globalizado (Borman, 2015; Ozigbo y Taiwo, 2014), por lo que resulta indispensable identificarlo, capturarlo, transferirlo y compartirlo. Además, este activo intangible se ha convertido en un factor de producción y es prioridad de las organizaciones en la sociedad del conocimiento, de ahí la importancia para el desarrollo de tecnologías, metodologías y estrategias que permitan su medición, creación y difusión (Rodríguez, 2006).

Davenport y Prusak (1998) señalan que el conocimiento constituye una mezcla en donde fluyen experiencias enmarcadas, valores e información contextual, y que en las organizaciones se inserta frecuentemente no solo en documentos o repositorios, sino también en rutinas, procesos, prácticas y normas organizacionales. Asimismo, este recurso es la base de aprendizajes e innovaciones, por lo que resulta una estrategia de gestión que, entre otras, "disminuye la redundancia de tareas y el número de errores en su ejecución, al aprovechar la experiencia existente dentro de la organización” (Medina, Medina y Nogueira, 2017, p. 17).

Por otra parte, la Organización para la Cooperación y el Desarrollo Económicos (OCDE, 2006) menciona que la innovación es la introducción de un producto (bien o servicio) nuevo o significativamente mejorado; un proceso; un método de comercialización, o un método organizativo en las prácticas internas de la empresa, la organización del lugar de trabajo o las relaciones exteriores.

La OCDE (2006) vincula la gestión del conocimiento con la innovación y apunta que la primera implica la apropiación, el manejo y la forma de colocar en común este conocimiento por la organización. De esta manera, enfatiza que se deben gestionar los vínculos externos y los flujos de conocimiento al interior de la empresa y establecer relaciones estrechas con otras compañías, clientes o instituciones de investigación. Nagles (2007) señala que, desde la perspectiva de la gestión del conocimiento, la innovación necesita desarrollar tres acciones fundamentales: reconocer las oportunidades de innovación, identificar los conocimientos relevantes que permitan la innovación, y generar soluciones sostenibles y rentables para la organización.

En la empresa familiar (EF) el conocimiento es una ventaja competiva (Leach, 1999), por lo tanto, la actitud emprendedora de los fundadores o de las generaciones subsecuentes juega un papel relevante como parte de la flexibilidad estratégica mediante las habilidades, conocimientos, capacidades, experiencia y valores, vistos como agentes detonantes, y que se forjan al inicio y durante su desarrollo (Rodríguez, Pico y Méndez, 2013). Esta actitud emprendedora en la EF también se relaciona, según Belausteguigoitia (2012), con la generación y aceptación de ideas en las organizaciones para enfrentar los cambios tecnológicos, regulatorios y demográficos, por lo que deben renovar constantemente sus prácticas, capacidades y actividades.

En las empresas pueden presentarse obstáculos para innovar (OCDE, 2006), entre ellos se encuentra el costo que implica la actividad y los factores asociados con el conocimiento, además, pueden tener éxito o no al tratar de alcanzar los objetivos que se habían fijado 
al introducir innovaciones, las cuales, a su vez, pueden tener efectos inesperados o adicionales con relación a los que se habían sustituido con su introducción. Hjalager (2010) indica que en el turismo las barreras para innovar tienen que ver con el acercamiento al conocimiento como, por ejemplo, patentes; a la difusión de la información y a la imitación.

Por otro lado, Martínez (2010) enfatiza que las ef son la esencia de la economía de mercado y las observa como la fuente generadora de un gran número de empleos y motor de la economía en casi todos los países. Por ello, la importancia del estudio de la ef se relaciona con la afirmación de $\mathrm{KPMG}^{1}$ México (2013) al mencionar que "más de 90 \% de las empresas que cotizan en la Bolsa Mexicana de Valores (BMv) tienen una clara representación familiar en el capital y en el control” (p. 5); además, se hace hincapié, con base en datos del Instituto Nacional de Estadística y Geografía (INEGI) publicados entre 2010 y 2012 citados por KPMG México (2013), que “las Micro, Pequeñas y Medianas Empresas (Mipymes), que básicamente corresponden al modelo familiar, suman $99 \%$ del total, con un promedio de 5.4 empleados" (p. 6). Por su parte, San Martín y Durán (2017, pp. 29-31) señalan que en México 83 \% de las empresas son familiares, correspondiendo 79.4 \% a los servicios, y que, por el tamaño de las EF, existen $57 \%$ de microempresas, $29 \%$ pequeñas, $11 \%$ medianas y $3 \%$ grandes.

En este contexto, el estudio de la ef hotelera es relevante en el destino turístico de Mazatlán, ya que, de acuerdo con cifras del INEGI (2016, p. 385 y 2017, p. 383), el número de hoteles de 2015 a 2016 fue de 138, mientras que la ocupación mediante la llegada de turistas a establecimientos de hospedaje en 2016 fue de 2154570 (INEGI, 2017, p. 388), observándose un crecimiento de $8.7 \%$ respecto a 2015 . Este incremento de la actividad hotelera es ante todo en la llegada de turistas nacionales provenientes del noreste del país, lo cual se debe a la operación de la autopista a Durango desde hace tres años.

El comportamiento de la actividad turística y la importancia de la EF hotelera en Mazatlán, Sinaloa, son elementos que se relacionan en este estudio de la integracion de conocimiento para la innovación. El destino turístico se caracteriza por ser tradicional. Al respecto, Herbert (2019), ${ }^{2}$ en una entrevista con el propietario de uno de los establecimientos de hospedaje, señala: "Las Flores, como muchos otros hoteles locales, es una empresa familiar. Eso distingue a Mazatlán de otros destinos de gran turismo mexicano: aunque han llegado franquicias internacionales, una importante presencia del empresariado autóctono se mantiene..." (s. p.).

Por lo anterior, el objetivo es identificar la integración de conocimientos para la innovación de procesos (IP) y organizativa (IO) en la EF hotelera de Mazatlán, Sinaloa, en el entendido de que el conocimiento es un recurso clave que procede de distintas fuentes y vínculos internos y externos y coadyuva con la innovación en un mercado globalizado. De esta manera, un cuerpo teórico de conceptos y teorías orienta y soporta la metodología y

${ }^{1}$ KPMG es la sigla conformada a partir de las iniciales de los apellidos de los principales miembros fundadores de la firma: $K$ de Piet Klynveld, $P$ de William Barclay Peat, $M$ de James Marwick y $G$ de Reinhard Goerdeler. Esta firma fusiona compañías de servicios profesionales en el mundo.

${ }^{2}$ El texto es un reportaje sobre el destino turístico en una revista en línea. 
el instrumento diseñado (OCDE, 2006; Choo, 1998; Hjalager, 2010; Jacob y Aguiló, 2008). Los resultados que se presentan corresponden al proyecto de investigación "Determinantes de la innovación y política turística en el destino Mazatlán, Sinaloa” (DIPTDMS).

Este documento se estructura de un cuerpo teórico denominado el conocimiento para la innovación y ef hotelera, así como la integración de conocimiento y la innovación; la metodología utilizada; los resultados obtenidos y las conclusiones.

\section{Conocimiento e innovación en la empresa familiar hotelera}

El conocimiento en la organización es un bien intangible, que se caracteriza por su renovación y que adquiere valor para la economía al compartirse con otras personas internamente, pero también con suministradores o clientes (Hernández, 2016). Suppiah y Sandhu (2011) citados por Bañegil, Barroso y Sanguino (2013) mencionan que el "conocimiento es reconocido como el recurso distintivo único y exclusivo, así como el diferenciador clave y crucial para cualquier organización para mantener su ventaja competitiva" (p. 74). El conocimiento en la organización es un proceso que incorpora valores, actitudes y creencias y que permite conocer su entorno e intervenir dinámicamente en este, con base en su experiencia (curva de aprendizaje) y sus habilidades (Fontalvo, Quejada y Puello, 2011). Por último se tiene que el conocimiento se crea con los datos e información al compararse, analizar las consecuencias, buscar conexiones y hablar con otros acerca de la información recibida (Sequeira y Marques, 2011).

Por otra parte, la innovación es la conversión de ideas y conocimientos en productos, procesos o servicios mejorados para el mercado, satisfaciendo así las necesidades de los ciudadanos, empresas y administraciones públicas (Afuah, 1999; Corma, 2011). Mathison, Gándara, Primera y García (2007) señalan que la innovación constituye "la capacidad para transformar los procesos empresariales y crear organizaciones más competitivas, ágiles y eficaces" (p. 69).

Hjalager (2010) enfatiza que la IP en el turismo se refiere a las iniciativas que apuntan a la escalada de la eficiencia, la productividad y el flujo. La inversión en tecnología se convierte en la principal fuente, y generaría mayor eficiencia si se combina con otra estrategia y medidas de gestión, como el desarrollo de competencias y la gestión de recursos humanos. Algunos ejemplos serían la rapidez en la preparación de alimentos o la mejora en el servicio de huéspedes.

La Io, de acuerdo con Jacob y Aguiló (2008), estimula mejorar la productividad y la calidad de los servicios turísticos en virtud de que los cambios organizativos coadyuvan en la motivación de innovaciones para enfrentarse a nuevos desafíos y dificultades. En tanto Hjalager (2010) dice que las innovaciones gerenciales llevan a formas de organización distintas en donde la colaboración interna, la dirección y la capacitación de personal se conjuntan, además de la creación de carreras y la compensación del salario. Se hace énfasis en el reto que tiene la empresa turística para desarrollar métodos que le permitan retener al personal y su satisfacción laboral, mantener flexibilidad y control de costos, cuidar los conocimientos y el activo de competencia. 
En relación con el concepto de EF se menciona que es una organización controlada y operada por los miembros de una familia (Belausteguigoitia, 2012; Leach, 1999; Rodríguez et al., 2013). Lorenzo y Núñez-Cacho (2012) plantean que existen tres puntos en común en las definiciones de EF: la propiedad, que se refiere al porcentaje de acciones o control efectivo de la empresa; la gestión, que atiende el desempeño de tareas ejecutivas y el nombramiento de ejecutivos, normalmente personas de la familia; $y$, por último, la continuidad, que alude a las generaciones familiares implicadas en la gestión de la empresa.

La EF hotelera se relaciona con el turismo por sus implicaciones y servicios de alojamiento que brinda a esa actividad, convirtiéndose en una unidad básica con un papel esencial en los destinos turísticos (Barron y Baum, 2007). Al respecto, el estudio de Peters y Buhalis (2004) sobre la planificación estratégica y la necesidad de educación y entrenamiento en las EF hoteleras da cuenta de resultados que tienen impacto en la innovación, ya que el empresario se ve a sí mismo y a su familia como la principal fuerza motriz de la actividad de innovación, dejando de lado a los empleados; por otra parte, otros hallazgos que se obtuvieron reflejan que los clientes y las ferias de turismo son impulsores externos de la innovación.

Por lo anterior, el conocimiento para la innovación en la EF hotelera se entiende como un recurso intengible que se procesa a través de valores, actitudes y creencias, el cual permite la interpretación del entorno y se interviene dinámicamente, además de ser la base de los aprendizajes e innovaciones, por lo que resulta una estrategia de gestión.

\section{La integración de conocimiento y la innovación}

En este artículo, la integración de conocimiento y la innovación en las EF hoteleras se relaciona con los enfoques teóricos de la agencia en la organización, el de recursos y capacidades, el capital intelectual, los relativos a gestión del conocimiento y, con ello, el de uso de la información en las organizaciones y los vínculos de conocimiento. En este sentido, los antecedentes de investigaciones que se mencionan a continuación llevan a demostrar cada perspectiva teórica.

En la teoría de la agencia se encuentra el aspecto positivo de la agencia, sobre lo cual Charreaux (2000) comenta que el conocimiento es relevante en el desempeño organizacional y de suyo en la eficiencia al contribuir en la toma de decisiones, por lo que existe un costo al transferir conocimiento entre actores; el ideal es encontrar el menor costo y ponerlo a disposición de los tomadores de decisiones. Al respecto, Hülsbeck et al. (2011) citado por Steeger y Hoffmann (2015) señalan que las EF tienen dificultades para utilizar su ventaja potencial, subrayan que los problemas de agencia en ellas son mayores que en los negocios no familiares, resultado de la concentración en la propiedad, teniendo como consecuencia el impacto innovador a la inversa. En el estudio de estos autores una de sus conclusiones es la aversión para enfrentar el riesgo a innovar en la EF.

De acuerdo con Ochoa, Prieto y Santidrián (2012), la teoría de los recursos y capacidades contempla la heterogeneidad empresarial y la construcción de la estrategia sobre 


\section{Conocimiento para la innovación en la empresa familiar hotelera}

Caso: destino turístico Mazatlán

los recursos de los que dispongan, así como la obtención de mayor renta y eficiencia. Los recursos provienen desde el producto de su historia "suerte y decisiones pasadas, sobre los que puede sustentar una ventaja competitiva y en la medida en que esta ventaja competitiva sea sostenible, le proporcionará una renta a largo plazo" (Ochoa, Prieto y Santidrián, 2012, p. 36). En este enfoque teórico, Lorenzo y Núñez-Cacho (2012) y Sirmon y Hitt (2003) identifican los recursos y las capacidades de la EF como un potencial, además de hacer énfasis en su desarrollo en la búsqueda de ventajas competitivas. Sirmon y Hitt (2003) concluyen en su investigación que el capital humano, y con ello el conocimiento, es de los recursos más importantes para una EF; es intangible, socialmente complejo y difícil de imitar, lo cual la conduce a crear una ventaja competitiva, sin embargo, este capital humano en ocasiones es inaccesible fuera de la familia, por lo que se requiere de una gestión efectiva de los recursos.

La perspectiva teórica del capital intelectual se refiere a la acumulación de conocimiento en una organización y que le aporta valor cognitivo. Este valor se compone de activos intangiles, que en combinación con los otros capitales físicos y financieros proporcionan ventajas competitivas en el mercado (Bueno, Salmador y Merino, 2008). Estudios y propuestas en la hotelería se han realizado desde este enfoque. Al respecto, Gazzera (2014), en la conjunción de la perspectiva de la gestión del conocimiento y del capital intelectual, propone el concepto de gerencia del conocimiento en la organización, cuya función radica en gerenciar los activos intangibles que permitan mantener y fortalecer la competitivdad de la empresa y argumenta que la distinción organizacional actualmente reside en la capacidad de combinar fuentes internas y externas del conocimiento para aprovechar las oportunidades comerciales.

Otra propuesta es la teoría dinámica del capital intelectual de Millán, Gómez y Vargas (2017) que contribuya a incrementar la competitividad en la hotelería. Los autores destacan que la limitada capacidad para aprender, innovar y reorganizarse se debe "a falta de sistematización de la información generada a partir de sus relaciones, a la demanda de implementación de tecnologías de la información que hagan eficientes sus procedimientos y a la alta rotación de personal” (p. 102), lo cual provoca que se pierdan conocimientos y experiencia. La concreción de la propuesta es transitar del capital intelectual estático que contiene los activos de los capitales humano, relacional y estructural hacia el capital intelectual dinámico mediante la aplicación sistemática de los activos del capital intelectual en los niveles administrativo, tecnológico y de información. Esto conduce a la competitividad e innovación.

En el enfoque teórico de la gestión del conocimiento, según Medina, Medina y Nogueira (2017), esta se entiende como un proceso que "promueve la generación, colaboración y utilización del conocimiento para el aprendizaje organizacional e innovación, con el que se genera nuevo valor y se eleva el nivel de competitividad en aras de alcanzar los objetivos organizacionales con eficiencia y eficacia" (p. 17).

La gestión del conocimiento incorpora ventajas a la organización, ya que incrementa la capacitación y la profesionalización de las personas y sus roles laborales, integra diferentes 
puntos de vistas de las distintas áreas de la empresa y de enfoques complementarios, genera nuevas formas de gestión y de operatividad, reduce costos y tiempos de entrega o fabricación, aumenta la innovación, entre otras (Contreras y Tito, 2013).

El soporte teórico en la gestión del conocimiento desde los modelos son citados por Farfán y Garzón (2006) como sigue: modelo de Bueno (2002); modelo Andersen (1999); proceso de creación del conocimiento de Nonaka y Takeuch (1995), que se da en un ciclo de socialización, exteriorización, combinación e interiorización; Knowledge Management Assessment Tool (KMAT), cuyos componentes son liderazgo, cultura, tecnología y medición; modelo de gestión del conocimiento de KPMG Consulting de Tejedor y Aguirre (1998); modelo Gopal \& Gagnon (1995); administración del conocimiento, administración de la información, administración del aprendizaje y modelo dinámico de creación de conocimiento, en donde se relacionan mercado y estrategia, estructura y procesos, conocimiento y sistemas con las personas y la motivación.

Hernández (2016) señala que la formulación de una estrategia adecuada para la gestión del conocimiento contribuye con lo siguiente: proponer una buena práctica; mejorar la comprensión de la actividad de gestionar el conocimiento en la organización; articular la actividad empresarial e identificar beneficios potenciales; obtener el compromiso de la alta dirección; lograr recursos para la implementación del sistema; ofrecer un plan claro y comunicable acerca de dónde se está, a dónde se quiere llegar y cómo se planifica llegar hasta ahí, y dar una base para medir el progreso.

Nagles (2007) plantea el esquema de gestión del conocimiento a partir de tres dimensiones: las barreras para su integración, la transferencia de conocimiento y las acciones para una efectiva gestión. En lo que se refiere a la transferencia de conocimientos, la divide en dos, primero en los elementos del proceso: adquisición y apropiación de conocimiento, solución de problemas, implementación e integración de soluciones, y experimentación y generación de prototipos; y, segundo, en proceso de transferencia, integración de nuevos conocimientos, localización y adaptación de conocimientos, adecuación de sistemas en la organización y diseño de nuevos productos, servicios y procesos. Medina et al. (2017) proponen la cadena de valor del conocimiento que tiene su alcance en la adquisición, la organización, la divulgación, el uso y la medición, esto último ya con el producto o servicio, y la detección de nuevas necesidades de información.

Hjalager (2010) menciona cuatro procesos de búsqueda y adquisición de conocimiento para la innovación en el turismo: el conocimiento incorporado; las competencias y el conocimiento basado en recursos; el conocimiento localizado y, por último, la investigación basada en el conocimiento.

Hallin y Marnburg (2007) argumentan que en el caso de la hotelería resulta relevante la incorporación de estrategias para la gestión del conocimiento, algunas razones se deben a implicaciones que se tienen: por un lado, el servicio de alojamiento con el de alimentos y bebidas, además de la gama de colaboradores que intervienen: centro de convenciones, agencias de viaje en línea, turoperadores, entretenimientos y tiendas con diversos productos, amén de la relación directa entre empleados y visitantes. 
Dos Anjos, Flores, Gadotti y Domareski (2011) indican que las contribuciones de la gestión del conocimiento a las empresas turísticas se tienen en Formica y Xiang (2007), Yang y Wan (2004), Yang (2004) y Yang (2008). Estas investigaciones hacen un análisis en compañías aéreas, agencias de viajes y hoteles. Las aportaciones aparecen en los ámbitos de la gestión estratégica, el conocimiento del consumidor, las prácticas de la gestión del conocimiento, el aprendizaje organizacional y el estímulo para compartir el aprendizaje individual y organizacional.

En el enfoque del uso de la información dentro de la teoría de gestión del conocimiento se distinguen tres formas, de acuerdo con Choo (1998): la de la percepción, que permite la interpretación; la de la creación de conocimiento, que convierte la información; y la de la toma de decisiones, en donde se analiza la información. En la forma de creación de conocimiento se concentra la idea de la organización que aprende, la creación se logra a través de la relación sinérgica entre conocimiento tácito y explícito y el diseño de procesos sociales que llevan al nuevo conocimiento; el autor enfatiza que al especializarse en la conversión de conocimientos se impulsa la innovación y el desarrollo de nuevos productos y servicios.

Respecto al enfoque teórico mencionado anteriormente, Borman (2015) señala que en las organizaciones hoteleras la inversión en gestión del conocimiento es relevante ya que lleva a un mejor rendimiento, por lo que no debería existir brecha para la captación de información en virtud de que esta permite una mejor planificación, toma de decisiones y la formulación de políticas.

En relación con la vinculación de conocimiento, Choo (1998) se refiere a la creación de conocimiento en la organización no de una manera aislada sino como colaboración de los miembros en lo interno y en sociedades con otras organizaciones, así es como Badaracco citado por Choo (1998) acuña el concepto de vínculos de conocimiento, para lo cual establece cuatro rangos: el primero es que el objetivo central de los vínculos es aprender y crear conocimiento, que se interesa por desarrollar capacidades en un plazo más largo; el segundo alude a la intimidad de los vínculos de conocimiento, lo que implica que los asociados aprendan, creen o fortalezcan las capacidades especializadas, y se requiere que el personal trabaje íntimamente; el tercero corresponde a la diversidad de vínculos con los asociados, entre compradores y proveedores, investigadores universitarios y compañías, sindicatos y sus empresas; y el cuarto es el potencial estratégico de los vínculos de conocimiento que aumentan las capacidades básicas de la organización. Ante ello, el conocimiento estratégico de la organización se encuentra en sus capacidades para generar conocimiento a través del tiempo y a largo plazo.

En conclusión, la distinción de adquisición de conocimientos en el turismo de Hjalager (2010), al relacionarse con los vínculos de conocimiento para la organización que mencionan la OCDE (2006) y Choo (1998), se aprecia en el cuadro 1. 
Cuadro 1. Adquisición de conocimiento para la innovación en el turismo y forma de vinculación de la organización

\begin{tabular}{|c|c|c|}
\hline $\begin{array}{l}\text { Adquisición de } \\
\text { conocimiento para } \\
\text { la innovación }\end{array}$ & Situación & $\begin{array}{c}\text { Vínculo de } \\
\text { conocimiento }\end{array}$ \\
\hline $\begin{array}{l}\text { Conocimiento } \\
\text { incoporado }\end{array}$ & $\begin{array}{l}\text { En su incorporación son fundamentales las cadenas de } \\
\text { negocios o redes que son relevantes para la transferencia de } \\
\text { conocimiento en el turismo. La crítica que se menciona es que } \\
\text { la estadarización se convierte en un obstáculo para el espíritu } \\
\text { innovador de las empresas. }\end{array}$ & Exterior \\
\hline $\begin{array}{l}\text { Las competencias y } \\
\text { conocimientos basados } \\
\text { en recursos }\end{array}$ & $\begin{array}{l}\text { El conocimiento significativo está disponible en la empresa y } \\
\text { en los integrantes, como tácito; ante ello, es necesario que } \\
\text { sea explicitado, entendido adecuadamente, interpretado, } \\
\text { restaurado y adaptado a las innovaciones específicas, pero } \\
\text { también recodificado. En este escenario se enfatiza que los } \\
\text { recursos humanos, y con ello el conocimiento y la capacidad } \\
\text { de innovación, se caracterizan por la migración, por lo que } \\
\text { son parámetros que deben estarse indagando en el mercado } \\
\text { laboral. }\end{array}$ & Interior \\
\hline $\begin{array}{l}\text { El conocimiento } \\
\text { localizado }\end{array}$ & $\begin{array}{l}\text { En este escenario de fuente de conocimiento, Hjalager observa } \\
\text { al destino turístico como un depósito de competencias y } \\
\text { conocimientos, únicos e inimitables, de las empresas y que } \\
\text { permiten el desarrollo de productos y servicios. Además hace } \\
\text { hincapié en conocimientos intersectoriales con que cuentan } \\
\text { los destinos. }\end{array}$ & $\begin{array}{l}\text { Exterior para la } \\
\text { empresa } \\
\text { Interior para el destino }\end{array}$ \\
\hline $\begin{array}{l}\text { Conocimiento basado } \\
\text { en la investigación }\end{array}$ & $\begin{array}{l}\text { Participación de la academia. Hjalager menciona que los } \\
\text { recursos que se desginan para este tipo de fuente son } \\
\text { escasos; además de la necesidad de empleo de personal bien } \\
\text { educado de cara a la alta rotación de personal y un bajo nivel } \\
\text { educativo general en la actividad. }\end{array}$ & Exterior \\
\hline
\end{tabular}

Fuente: Elaboración propia con base en Hjalager (2010), Choo (1998) y ocDE (2006).

\section{Metodología}

Esta comunicación tiene como base el proyecto de investigación DIPTDMS, que focalizó la IP e 10 en los establecimientos de servicio turístico, cuyos resultados arrojaron, entre otras, la línea de investigación Innovación en la empresa familiar hotelera. En este sentido, la integración de conocimiento es una variable que se abordó como propulsora de la actividad de innovación en las organizaciones y, en este caso, en las EF hoteleras.

El objetivo de identificar la integración de conocimientos para la IP e Io en la EF hotelera de Mazatlán, Sinaloa, llevó a decidir el enfoque cuantitativo a partir del fundamento teórico de cuatro dimensiones: las actividades de innovación, las fuentes de información de libre 


\section{Conocimiento para la innovación en la empresa familiar hotelera \\ Caso: destino turístico Mazatlán}

acceso o gratuitas, la adquisición de conocimiento y tecnología por compra y, por último, la cooperación, y con ello la vinculación interna o externa del conocimiento (OCDE, 2006; Choo, 1998). De esta manera, se analizan descriptivamente los promedios alcanzados en las dimensiones y categorías que son fuente y vínculo del conocimiento para la IP e IO.

Las dimensiones señaladas se definen a continuación, de acuerdo con la OCDE (2006):

1. Las actividades de innovación son el conjunto de actuaciones científicas, tecnológicas, organizativas, financieras y comerciales que conducen a la introducción de innovaciones (p. 25).

2. Las fuentes de información de libre acceso o gratuitas se entienden como la información de difusión libre, que no requiere la compra de tecnología o derechos de propiedad intelectual; además incluye la investigación y desarrollo que se realiza dentro de la empresa, así como el conocimiento que se transmite por organizaciones del mismo grupo (p. 91).

3. La adquisición de conocimientos y tecnología se establece por la compra de conocimiento externo y de tecnología que se encuentra en bienes de equipo o servicios (p. 92).

4. La fuente de cooperación se entiende como la cooperación activa que se tiene con otras empresas o instituciones públicas de investigación (p. 92).

Además, al indagar estudios similares a este objeto y formas metodológicas de abordarlos se encontró que el conocimiento y la innovación en la EF han sido poco investigados (Lorenzo y Núñez-Cacho, 2012); así, estos autores analizan la innovación y aplican el modelo de inercias de Rumelt a la EF; por su parte, Steeger y Hoffmann (2015), a través del modelo Tobit, realizan una investigación de tipo transversal, y Borman (2015) estudia la empresa hotelera en Ghana a partir del sistema de gestión del conocimiento y las ventajas competitivas mediante el enfoque cuantitativo.

Por otro lado, esta investigación es transversal, ya que la recolección de datos se hizo en un mes (julio de 2017) ${ }^{3}$ a partir de la aplicación de un cuestionario elaborado y adaptado con base en el Manual de Oslo (OCDE, 2006); cabe destacar que este documento contempla la importancia de la innovación en los sectores de servicio y con baja densidad en investigación y desarrollo y contiene preguntas dicotómicas. En la primera parte del instrumento se inquirió sobre información general de los establecimientos: la asociación a la que pertenece el establecimiento de hospedaje, característica del hotel en estrellas, número de empleados que laboran, porcentaje de usuarios tanto nacionales como extranjeros que se hospedan, y si se realizan actividades innovadoras y sus tipos.

En la segunda parte se preguntó primero por la dimensión actividades de innovación que contiene nueve ítems (investigación y desarrollo interno; adquisición de investigación y desarrollo externa; introducción, desarrollo, ensayos y métodos de procesos; planificación

\footnotetext{
${ }^{3}$ Este proyecto fue financiado por el Programa para el Desarrollo Profesional Docente, para el Tipo Superior (Prodep), por lo que se ajusta a un periodo de un año para su diseño, ejecución y finalización. Además, las fechas de aplicación del cuestionario fueron concertadas con los gerentes de los establecimientos con base en su disposición de tiempo.
} 
e introducción de nuevos métodos de organización; adquisición de equipos y máquinas; adquisición de terrenos; formación de capital humano; gastos corrientes e innovación; e inversión en capital fijo en innovación). Después se abordó la dimensión integración de conocimiento y tecnología a la IP e 10 con tres variables: fuentes de información de libre acceso o gratuitas; adquisición de conocimiento y tecnología por compra y, la última, por cooperación activa en innovación.

En cuanto al caso estudiado, el destino turístico Mazatlán se ubica en el estado de Sinaloa, al noroeste de la república mexicana, en donde la actividad económica preponderante es el turismo que se caracteriza por ser de sol y playa. Los establecimientos hoteleros en el destino suman 183 (INEGI, 2017), y los integrados a las dos asociaciones que existen son 40 que se compone de cadenas hoteleras, corporativos y EF, 13 corresponden a establecimientos registrados en la Asociación de Hoteles y Moteles Tres Islas de Mazatlán (АНMтIM) y 27 a la Asociación de Hoteles y Empresas Turísticas de Mazatlán (АHETM). De esta población, la muestra fue de 15 hoteles que cubrían los criterios que se consideraron desde el diseño original del proyecto DIPTDMs. Ahora bien, al ampliar la línea de investigación Innovación en la empresa familiar, se seleccionaron cuatro EF hoteleras que se distribuyeron de manera proporcional en función de la asociación a la que pertenecen y se tuvieron en cuenta los criterios siguientes:

a) Los informantes seleccionados para el cuestionario fueron los gerentes generales de los establecimientos.

b) Ubicación geográfica: hoteles que se encuentran sobre la playa o que se separan de esta mediante una calle.

c) Establecimientos integrados en alguna de las dos asociaciones.

d) Establecimientos con funcionamiento en el mercado superior a los tres años y con una sucesión como mínimo.

e) Las actividades de innovación se refieren solo a IP e 10.

Asimismo, se utilizan variables nominales que contienen categorías no jerárquicas ni ordenadas para efectos de la evaluación y de comparación como la media aritmética, la cual indica el valor promedio y de frecuencia relativa. En el análisis entre variables e indicadores que lo integran, se busca identificar y explicar, en función de los avances mostrados, que la integración de conocimiento ha tenido impacto en las actividades de innovación.

\section{Resultados}

Los resultados se presentan en tres segmentos: el primero se refiere a la información general de los establecimientos informantes, después se señalan las actividades de innovación y, por último, se describen los hallazgos que tienen que ver con la integración de conocimiento a las IP e IO. 


\section{Conocimiento para la innovación en la empresa familiar hotelera \\ Caso: destino turístico Mazatlán}

\subsection{Información general de los establecimientos}

Los datos de los hoteles informantes arrojaron los hallazgos siguientes: los cuatro hoteles son de cuatro estrellas, y por el número de empleados que los integran son: $25 \%$ de 50 a $99,50 \%$ de 100 a 249 y $25 \%$ de 250 a 999. El origen de sus usuarios es: $25 \%$ (70\% nacionales y $30 \%$ internacionales), $25 \%$ (80 \% nacionales y $20 \%$ internacionales), $25 \%$ (85 $\%$ nacionales y $15 \%$ internacionales) y $25 \%$ (91\% nacionales y $9 \%$ internacionales). Los cuatro establecimientos informaron que han realizado innovaciones en las tipologías de producto o servicio, proceso, organizativas y de mercadotecnia.

\subsection{Actividades de innovación}

Los establecimientos señalaron (figura 1) que $100 \%$ planifica e introduce nuevos métodos de organización y realiza actividades de investigación y desarrollo interno, 25 \% adquirió terrenos y cuenta con gastos corrientes en innovación. En este sentido, las actividades de innovación que se efectúan se concentran en investigación y desarrollo interno con una formación de capital humano de $75 \%$.

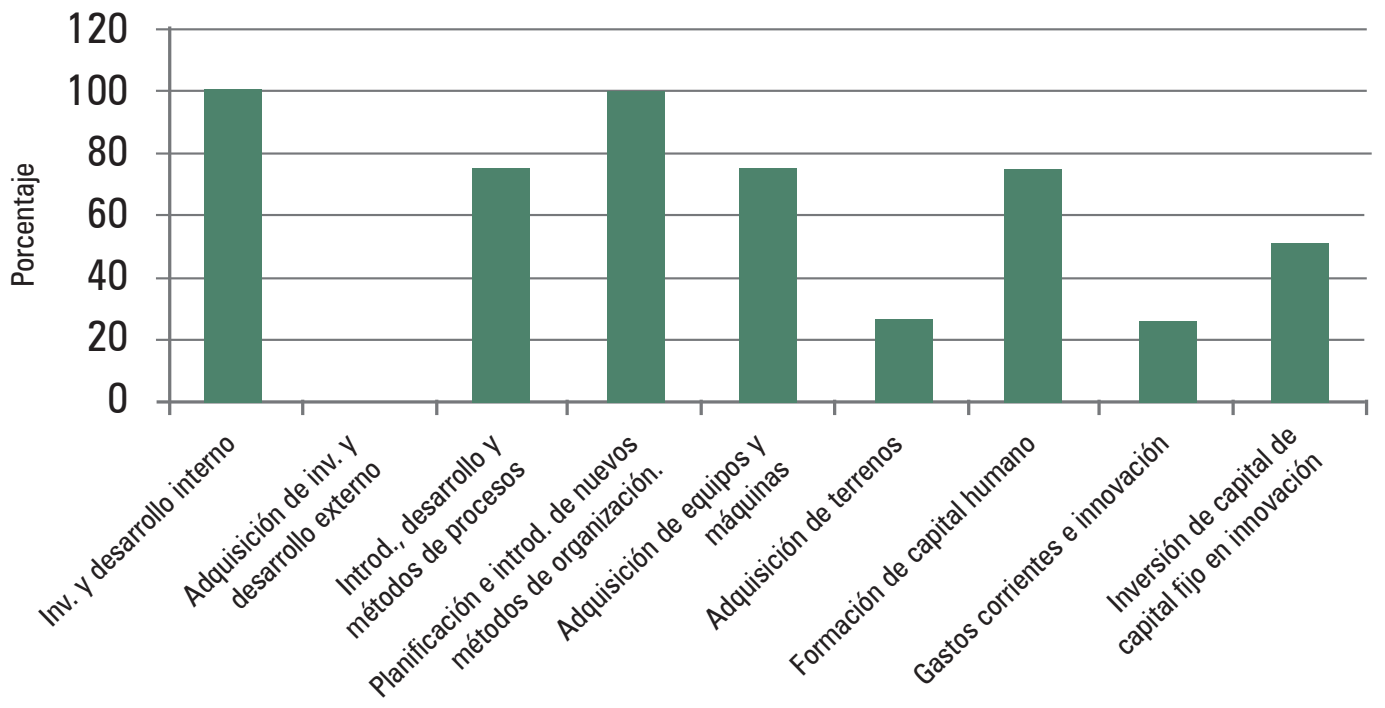

Fuente: Elaboración propia.

Figura 1. Actividades de innovación

\subsection{Integración de conocimiento a la innovación de procesos y organizativa gratuitas}

En esta dimensión se integran las fuentes gratuitas, por compra y por cooperación. De esta manera, la integración de conocimiento a través de estas fuentes (figura 2) demuestra que en las IP por compra arroja un promedio de $61.2 \%$, mientras que por cooperación 


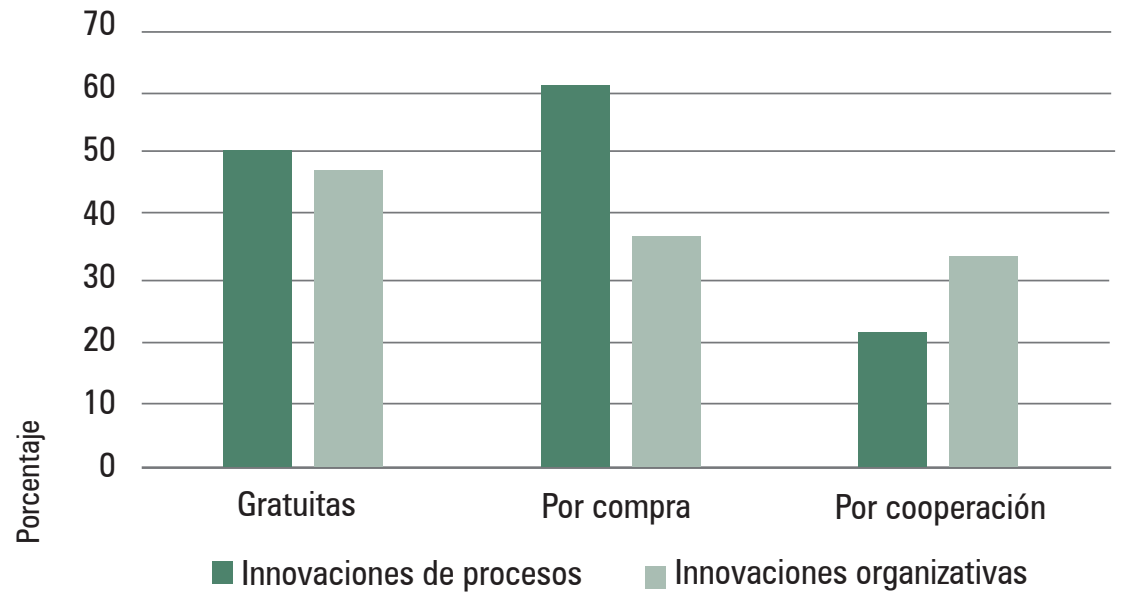

Fuente: Elaboración propia.

FiguRA 2. Integración de conocimiento por fuentes: gratuitas, por compra y por cooperación

es de $22 \%$. En las 10, las gratuitas reflejan $47.4 \%$, y las de cooperación $33.7 \%$. Esta información visibiliza que la cooperación es escasa y no se aprovechan suficientemente las relaciones, por ejemplo, con las instituciones educativas y de investigación para incorporar conocimiento.

Por otra parte, en relación con la integración de conocimiento y tecnología al establecimiento mediante fuentes gratuitas (figura 3), se observa en las IP a las internas con un promedio de $66.7 \%$, como el más alto; en tanto, el menor se tiene en las fuentes de información general con $39.3 \%$. En las Io se observa que las fuentes comerciales y de mercados exteriores son las más altas con 60 \% y las menores recaen en fuentes que dependen del sector público con $33 \%$.

Los informantes señalaron que estas fuentes las obtienen primordialmente de expertos, investigación y desarrollo interno, clientes y redes informales, y que la experiencia del personal es un factor relevante en la integración de conocimiento.

Respecto a la integración de conocimiento y tecnología por compra (figura 4), en las IP los resultados arrojan que $45 \%$ integran conocimiento a través de fuentes comerciales y mercados exteriores, mientras que $50 \%$ de fuentes de información y general. En las ı, $31.2 \%$ obtiene conocimiento mediante fuentes de información generales.

Los informantes comentaron que la fuente principal para la integración de conocimiento por compra son los proveedores y las ferias y exposiciones; es decir, se manifiesta la confianza en el conocimiento probado, pero también en el que se demuestra con una experiencia con resultados positivos, como el caso del exhibido en exposiciones.

Por último, sobre la integración de conocimiento y tecnología por cooperación (figura 5) se observa que, para las IP, 35 \% proviene de fuentes comerciales y mercados exteriores, 
Conocimiento para la innovación en la empresa familiar hotelera

Caso: destino turístico Mazatlán

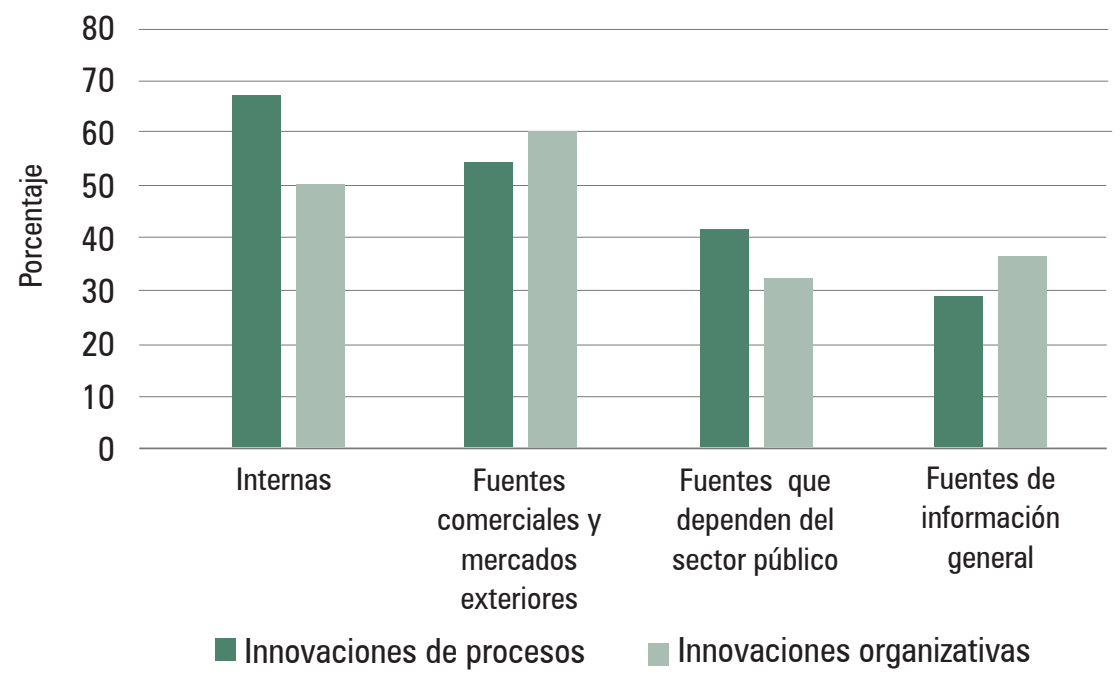

Fuente: Elaboración propia.

FiguRa 3. Integración de conocimiento y tecnología con fuentes de información gratuitas

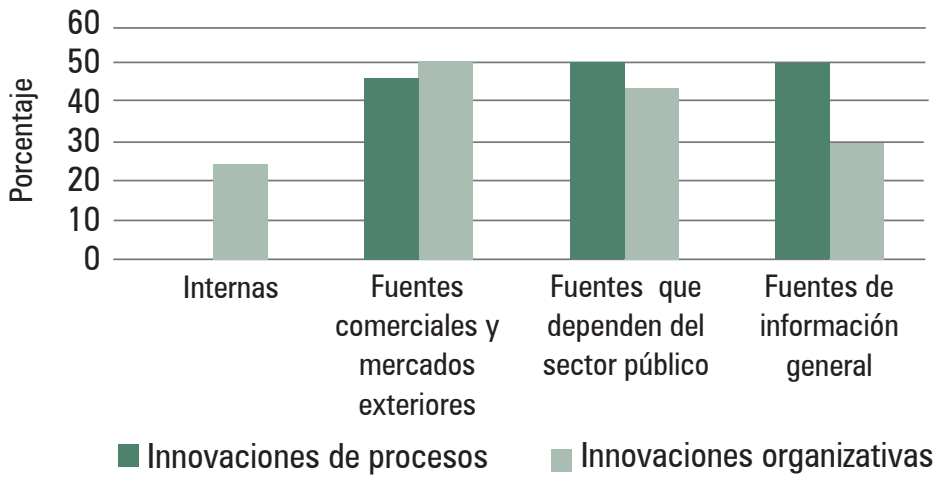

Fuente: Elaboración propia.

FIGURA 4. Integración de conocimiento y tecnología por compra

mientras que $6.2 \%$ de fuentes que dependen del sector público. Las Io lo obtienen en $50 \%$ de fuentes internas, y en $7 \%$ de fuentes que dependen del sector público.

En estas fuentes destacan las que se adquieren de los competidores y de los clientes/ usuarios. Este hallazgo denota que las recomendaciones y la confianza en los clientes-visitantes son un factor relevante en la integración de conocimiento de frente a la satisfacción del servicio y con la posibilidad de que retorne al establecimiento. 


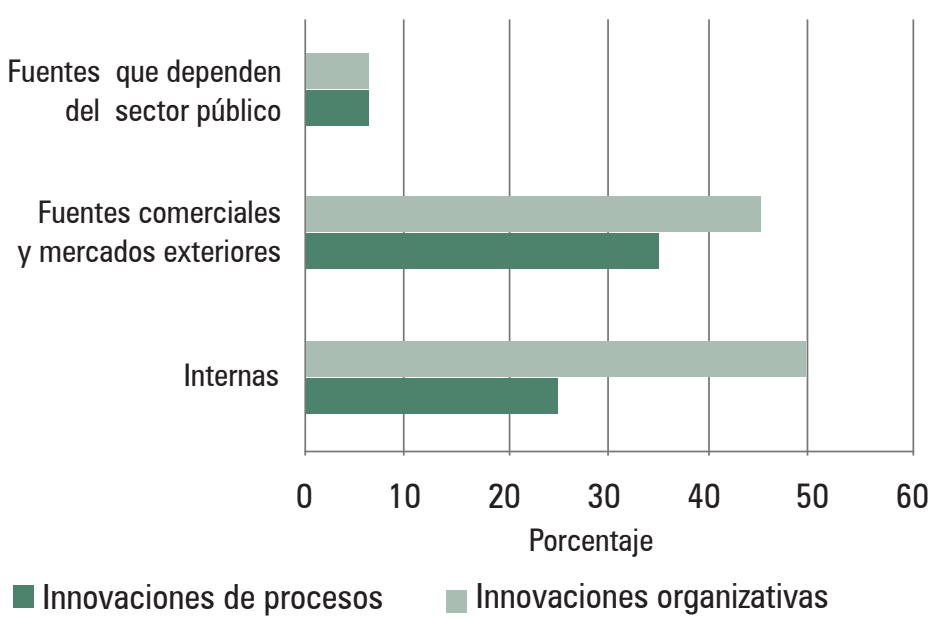

Fuente: Elaboración propia.

Figura 5. Integración de conocimiento y tecnología por cooperación

\section{Análisis y discusión}

Las ef hoteleras estudiadas en el destino turístico Mazatlán, por el número de empleados, son medianas y grandes (INEGI, 2009), y ofrecen servicios de alojamiento a usuarios nacionales y extranjeros.

Las cuatro EF hoteleras que forman parte del estudio señalaron haber realizado actividades de innovación en los últimos tres años en las tipologías de producto o servicio, proceso, organizativas o de mercadotecnia, de acuerdo con la OCDE (2006); sin embargo, en la gama de actividades expuesta es significativo el resultado nulo de la adquisición de investigación y desarrollo externo; al respecto, Hjalager (2010) indica que en el turismo las barreras para innovar tienen que ver con el acercamiento al conocimiento, como por ejemplo patentes, y a la difusión de la información; además, este hallazgo se relaciona con la teoría de la agencia y las aportaciones de Steeger y Hoffmann (2015) al apuntar que la concentración en la propiedad en ocasiones no permite correr el riesgo para innovar en la EF debido al costo que representa.

En cuanto a las actividades de innovación, también se observa que la formación de capital humano no se presenta en el total de los establecimientos; este hallazgo se vincula con la teoría de los recursos y capacidades y con lo planteado por Sirmon y Hitt (2003), ya que el conocimiento se convierte en una ventaja competitiva de la organización que requiere de una gestión efectiva de los recursos. Asimismo se manifiesta una gerencia de los activos intangibles insuficientes para aprovechar las oportunidades comerciales (Gazzera, 2014).

Respecto a los vínculos de conocimiento, los hallazgos dan cuenta de que las EF hoteleras agencian información para crear conocimiento, teniendo el siguiente comportamiento: 
es medianamente significativo el resultado obtenido de fuentes gratuitas, por compra y por cooperación; por ejemplo, para la integración de información por compra para IP, cuyo resultado es el más alto, se adquiere sobre todo de empresas del mismo grupo y proveedores de equipos y materiales, es decir, internas y externas. En ıo, las fuentes gratuitas no alcanzan $50 \%$ en la más alta, y aquí se tiene que son las área de hospedaje, alimentos y bebidas, esparcimiento y diversión, y administración de la organización, principalmente, las que aportan la información con la que se crea conocimiento. Este comportamiento se liga con lo que menciona Badaracco (1991) citado por Choo (1998) a correr el riesgo de no observarse el potencial estratégico que proporcionan los vínculos de conocimiento y que aumentan las capacidades básicas de la organización.

De la relación entre las actividades de innovación y los resultados de la integración de conocimiento por fuentes: gratuitas, por compra y por cooperación, son medianamente aprovechadas las gratuitas y por cooperación para incorporar IP, lo que impacta en las actividades de introducción y desarrollo de nuevos métodos; esto es, las capacidades de la organización se manifiestan limitadas por la falta de sistematización de información generada a partir de sus relaciones (Millán, Gómez y Vargas, 2017), por ejemplo, con las universidades.

Por otro lado, en las EF hoteleras la priorización a vínculos externos (expertos, clientes y redes informales) aunados a internos, la experiencia del personal, da cuenta de que se tiene una perspectiva del proceso de transferencia, ya que se integran nuevos conocimientos, se localizan y adaptan esos conocimientos (Nagles, 2007), pero también un acercamiento al modelo dinámico de creación de conocimiento en donde se relacionan el mercado y la estrategia, estructura y procesos, conocimiento y sistemas con las personas y la motivación (Farfán y Garzón, 2006).

Además, es significativo el bajo promedio alcanzado en fuentes gratuitas, por compra y por cooperación tanto para IP como para Io en las que dependen del sector público, es decir, hay una escasa relación con este sector. Esto se vincula con el conocimiento estratégico y la capacidad como resultado de la calidad de la red externa de la organización, según Choo (1998).

\section{Conclusiones}

En la economía del conocimiento y en la globalización de los mercados el valor de este recurso intengible es relevante, ya que a través de su gestión estratégica y sistematización mediante su adquisición, organización, divulgación, uso y medición constante, se lograrán mantener o crear ventajas competivas e innovaciones en las organizaciones.

Al identificar la integración de conocimientos para la IP e Io en la ef hotelera de Mazatlán, Sinaloa, se confirma que el conocimiento es un recurso clave, y que sus fuentes y vínculos externos e internos deben gestionarse permanentemente. En este sentido, los resultados contribuyen en las perspectivas teóricas de gestión del conocimiento y del capital intelectual, además, en los estudios del turismo a través de las fuentes de conocimientos 
localizados y las competencias y conocimientos basados en recursos que han permitido actividades de investigación y desarrollo interno en cuatro ef hoteleras (Hjalager, 2010).

Las EF hoteleras que prestan servicios a usuarios nacionales y extranjeros del destino turístico Mazatlán no introducen investigación y desarrollo externo, es decir, evitan riesgos para innovar, por un lado, por el costo que representa y, por otro, por una limitada gerencia del conocimiento, lo que disminuye sus ventajas competitivas. Así pues, resulta recomendable que las ef formulen estrategias adecuadas de gestión del conocimiento (Hernández, 2016) como una buena práctica y para articular la actividad empresarial e identificar los beneficios potenciales.

Por otra parte, el rol del capital humano y el de los visitantes que se hospedan en las EF hoteleras son cardinales. Los primeros, con su contribución para el desarrollo de actividades de investigación interna, aunque la formación de este recurso no se da en la totalidad de las organizaciones; mientras que los visitantes se convirten en los principales proveedores de conocimiento, a los que se perfilan en la categoría de cooperación.

El conocimiento localizado (Hjalager, 2010) en estas EF hoteleras es un valor y una oportunidad en un destino tradicional, ya que sus competencias únicas contribuyen al desarrollo de los servicios de alojamiento que proporcionan en la actividad turística.

El alcance del estudio recae en cuatro EF hoteleras que se seleccionaron con base en criterios, por una parte teóricos para contar con resultados válidos y espaciales para asegurar los establecimientos que brindan el servicio turístico. Esto se convierte en una limitación, por lo cual es recomendable ampliar la muestra del estudio.

Por último, la continuación de la investigación se puede orientar a analizar la gestión del conocimiento a partir del modelo dinámico de creación de conocimiento o también el impacto del capital intelectual dinámico en las EF hoteleras.

\section{Fuentes consultadas}

Afuah, A. (1999). La dinámica de la innovación organizacional, el nuevo concepto para lograr ventajas competitivas y rentabilidad. México: Oxford.

Bañegil, T., Barroso, A. y Sanguino, R. (2013). La gestión del conocimiento en las empresas familiares. En W. Fernández, Nuevas investigaciones sobre la gestión de la empresa familiar en España (pp. 69-84). Barcelona: OmniaScience. Disponible en http:// dx.doi.org/10.3926/oms.03

Barron, P. y Baum, T. (2007). El alojamiento. En Ch. Cooper, J. Fletcher, A. Fyall, D. Gilbert y S. Wanhill, El turismo, teoría y práctica (pp. 459-498). Madrid: Síntesis.

Belausteguigoitia, I. (2012). Empresas familiares, dinámicas, equilibrio y consolidación. México: McGraw-Hill.

Borman, F. (2015). Assessing implementation of Knowledge Management Systems in hoteIs: The case of Ho, Ghana. African Journal of Hospitality, Tourism and Leisure, 4(2), 1-11. Recuperado de http://www.ajhtl.com/uploads/7/1/6/3/7163688/article11vol4(2)june_2015.pdf 
Bueno, E., Salmador, M. P. y Merino, C. (2008). Génesis, concepto y desarrollo del capital intelectual en la economía del conocimiento: Una reflexión sobre el Modelo InteIlectus y sus aplicaciones. Estudios de Economía Aplicada, 26(2), 43-63. Recuperado de http:/ /www.redalyc.org/articulo.oa?id=30113187003

Charreaux, G. (2000). La theorie positive de l'agence: positionnement et apports. En Revue d'économie industrielle, 92(2e), 193-214. Recuperado de https://doi. org/10.3406/rei.2000.1046

Choo, Ch. (1998). La organización inteligente. El empleo de la información para dar significado, crear conocimiento y tomar decisiones. México: Oxford University Press.

Contreras, F. y Tito, P. L. (2013). La gestión del conocimiento y las políticas públicas. Lima: Universidad María Auxiliadora. Recuperado de repositorio.uma.edu.pe/handle/ UMA/ 111

Corma, F. (2011). Innovación, innovadores y empresa innovadora. Madrid: Díaz de Santos.

Davenport, T. y Prusak, L. (1998). Working Knowledge: How Organizations Manage What They Know. Boston: Harvard Business School Press.

Dos Anjos, F. A., Flores, P., Gadotti, S. A. y Domareski, T. C. (2011). Contribuciones de la gestión del conocimiento a los servicios turísticos. Estudio en una agencia de viajes. Estudios y Perspectivas en Turismo, 20(3), 722-737. Recuperado de http:// www.redalyc.org/articulo.oa?id=180717583011

Farfán, D. Y. y Garzón, M. A. (2006). La gestión del conocimiento (Documento de investigación 29). Bogotá: Universidad del Rosario.

Fontalvo, T., Quejada, R. y Puello, J. (2011). La gestión del conocimiento y los procesos de mejoramiento. Dimensión Empresarial, 9(1), 80-87. Disponible en https://dialnet. unirioja.es/revista/16449/V/9

Gazzera, M. A. (2014). Los recursos humanos en la gerencia del conocimiento. Sector Hotelero, Patagonia, Argentina. Revista Electrónica CECIET, VI, 78-98.

Hallin, C. A. y Marnburg, E. (2007). Knowledge management in the hospitality industry: A review of empirical research. Tourism Management, 1-15. doi:10.1016/j.tourman.2007.02.019

Herbert, J. (25 de enero de 2019). El camino hacia Mazatlán. ¿Cómo se ha transformado uno de los grandes puertos mexicanos? Gatopardo, 197. Recuperado de https:// gatopardo.com/no-197-diciembre-2018-enero-2019/julian-herbert/

Hernández, V. (2016). La gestión del conocimiento en las organizaciones. Buenos Aires: Alfaomega Grupo Editor.

Hjalager, A. M. (2010). A review of innovation research in tourism. Tourism Management, 31, 1-12. Recuperado de https://www.academia.edu/5254430/Progress_in_Tourism_Management_A_review_of_innovation_research_in_tourism.

INEGI. (2009). Micro, pequeña, mediana y gran empresa. Estratificación de los establecimientos. Censos Económicos 2009. Instituto Nacional de Estadística y Geografía. Disponible en http://www.inegi.org.mx/est/contenidos/espanol/proyectos/censos/ ce2009/pdf/mono_micro_peque_mediana.pdf

INEGI. (2016). Anuario Estadístico y Geográfico de Sinaloa 2016. Instituto Nacional de Estadística y Geografía.

INEGI. (2017). Anuario Estadístico y Geográfico de Sinaloa 2017. Instituto Nacional de Estadística y Geografía. 
Jacob, M. y Aguiló, E. (2008). La innovación en el sector turístico: el caso de Baleares. Rotour/Revista de Ocio y Turismo, 1, 51-64.

KPMG México. (2013). Empresas familiares en México: El desafío de crecer, madurar y permanecer. Recuperado de https://assets.kpmg/content/dam/kpmg/mx/ pdf / 2016/12/Empresas\%20Familiares\%20en\%20M\%C3\%A9xico\%20el\%20desaf\%C3\%ADo\%20de\%20crecer\%20madurar\%20y\%20permanecer.pdf

Leach, P. (1999). La empresa familiar. Buenos Aires: Ediciones Garnica.

Lorenzo, J. D. y Núñez-Cacho, P. (2012). Inercia e innovación en la empresa familiar: una primera aproximación. Revista de Empresa Familiar, 2(2), 23-40. Recuperado de http://dx.doi.org/10.24310/ejfbejfb.v2i2.4027

Martínez, J. (2010). Empresas familiares, reto al destino. Buenos Aires: Ediciones Garnica.

Mathison, L., Gándara, J., Primera, C. y García, L. (2007). Innovación: Factor clave para lograr ventajas competitivas. Revista NEGOTIUM/Ciencias Gerenciales, 3(7), 46-83. Recuperado de http://www.redalyc.org/articulo.oa?id=78230705

Medina, D., Medina, A. y Nogueira, D. (2017). Procesos y factores claves en la gestión del conocimiento. Universidad y Sociedad, 9(2), 16-23. Recuperado de http://rus.ucf. edu.cu/index.php/rus

Millán, C., Gómez, M. del R. y Vargas, E. (2017). Gestión del capital intelectual (cI) dinámico en la hotelería, una reflexión teórica. Teoría y Praxis, 22, 85-112. Recuperado de http://www.redalyc.org/articulo.oa?id=456152576005

Nagles, G. (2007). La gestión del conocimiento como fuente de innovación. Revista Escuela de Administración de Negocios, 61, 77-87. Recuperado de http://www.redalyc. org/articulo.oa?id=20611495008

OCDE. (2006). Manual de Oslo. Madrid: Grupo Tragsa.

Ochoa, M. L., Prieto, M. G. y Santidrián, A. (2012). Una revisión de las principales teorías aplicables al capital intelectual. Revista Nacional de Administración, 3(2), 3548. Recuperado de http://investiga.uned.ac.cr/revistas/index.php/rna/article/ view/486/389

Ozigbo, N. y Taiwo, E. (2014). The dynamics of knowledge management practices for sustainable adoption in the nigerian hospitality industry. International Journal of Advanced Research in Statistics, Management and Finance, 2(1), 77-89. Recuperado de http://www.internationalpolicybrief.org/images/journals/Stat2.1/Stat7.pdf

Peters, M. y Buhalis, D. (2004). Family hotel Businesses: Strategic planning and the need for education and training. Education+Training, 46(8/9), 406-415. doi: $10.1108 / 00400910410569524$

Rodríguez, D. (2006). Modelos para la creación y gestión del conocimiento: una aproximación teórica. Educar, 37, 25-39. Recuperado de https://ddd.uab.cat/pub/educar/0211819Xn37/0211819Xn37p25.pdf

Rodríguez, P., Pico, B. y Méndez, F. J. (2013). Capacidad innovadora en la empresa familiar como área de oportunidad hacia el desarrollo de México. Economía, Sociedad y Territorio, XIII(43), 779-794. Recuperado de http://www.redalyc.org/articulo. oa?id=11127581007

San Martín, J. M. y Durán, J. (2017). Radiografía de la empresa familiar en México. Puebla: Fundación de la Universidad de las Américas. Recuperado de https:/ /www.udlap. $\mathrm{mx} /$ empresasfamiliares/assets/files/radiografiaEmpresaFamMex.pdf 


\section{Conocimiento para la innovación en la empresa familiar hotelera}

Caso: destino turístico Mazatlán

Sequeira, B. y Marques, J. F. (2011). Knowledge management in tourism organisations: Proposal for an analytical model. Discussion Papers-Spatial and Organizational Dynamics, 7. Recuperado de http://sapientia.ualg.pt/handle/10400.1/4580

Sirmon, D. y Hitt, M. (2003). Managing resources: Linking unique resources, management, and wealth creation in family firms. Entrepreneurship, Theory and Practice, 27(4), 339-358. doi: 10.1111/1540-8520.t01-1-00013

Steeger, J. y Hoffmann, M. (2015). Innovation and Family Firms: Departing From R\&D Mediations (WiSo-HH Working Paper Series, Working Paper 13). Universidad de Hamburgo. Recuperado de https://www.wiso.uni-hamburg.de/forschung/archiv/workingpaper/wpneuesdesign/wp27 